TRANSACTIONS OF THE

AMERICAN MATHEMATICAL SOCIETY

Volume 359, Number 5, May 2007, Pages 1959-1976

S 0002-9947(06)03941-9

Article electronically published on November 17, 2006

\title{
KAZHDAN GROUPS WITH INFINITE OUTER AUTOMORPHISM GROUP
}

\author{
YANN OLLIVIER AND DANIEL T. WISE
}

\begin{abstract}
For each countable group $Q$ we produce a short exact sequence $1 \rightarrow N \rightarrow G \rightarrow Q \rightarrow 1$ where $G$ has a graphical $\frac{1}{6}$ presentation and $N$ is f.g. and satisfies property $T$.

As a consequence we produce a group $N$ with property $T$ such that $\operatorname{Out}(N)$ is infinite.

Using the tools developed we are also able to produce examples of nonHopfian and non-coHopfian groups with property $T$.

One of our main tools is the use of random groups to achieve certain properties.
\end{abstract}

\section{INTRODUCTION}

1.1. Main statements. The object of this paper is to use a tool developed by Gromov to produce groups with property $T$ that exhibit certain pathologies. Our main result is a property $T$ variant of Rips' short exact sequence construction. We apply this to obtain a group with property $T$ that has an infinite outer automorphism group. In two further examples, we produce non-Hopfian and non-coHopfian groups with property $T$.

In Rip82, Rips gave an elementary construction which, given a countable group $Q$, produces a short exact sequence $1 \rightarrow N \rightarrow G \rightarrow Q \rightarrow 1$, where $G$ is a $C^{\prime}\left(\frac{1}{6}\right)$ group and $N$ is finitely generated. Rips used his construction to produce $C^{\prime}\left(\frac{1}{6}\right)$ presentations with various interesting properties, by lifting pathologies in $Q$ to suitably reinterpreted pathologies in $G$.

In Gro03, Gromov produced (random) groups with property $T$ that have graphical $\frac{1}{6}$ small cancellation presentations. The graphical $\frac{1}{6}$ small cancellation condition is a generalization of the classical $C^{\prime}\left(\frac{1}{6}\right)$ condition (see [LS77]) to presentations determined by labelled graphs. We refer to Section 2 and Oll03a for a discussion of this property.

The mixture of these two tools yields the following in Section 3 ,

Theorem 1.1. For each countable group $Q$, there is a short exact sequence $1 \rightarrow$ $N \rightarrow G \rightarrow Q \rightarrow 1$ such that

(1) $G$ is torsion-free,

(2) G has a graphical $\frac{1}{6}$ presentation,

Received by the editors September 27, 2004 and, in revised form, January 10, 2005.

2000 Mathematics Subject Classification. Primary 20F28, 20F06, 20E22, 20 P05.

Key words and phrases. Outer automorphism groups, property $T$, small cancellation, random groups.

This research was partially supported by NSERC grant.

(C)2006 American Mathematical Society Reverts to public domain 28 years from publication 
(3) $N$ has property $T$, and

(4) $G$ is finitely generated if $Q$ is, and $G$ is finitely presented if $Q$ is.

The graphical $\frac{1}{6}$ presentation retains enough properties of ordinary small cancellation theory to mix nicely with Rips' construction. However, we note that Theorem 1.1 cannot be obtained with $G$ an ordinary $C^{\prime}\left(\frac{1}{6}\right)$ group, since finitely presented $C^{\prime}\left(\frac{1}{6}\right)$ groups act properly on a CAT(0) cube complex by Wis04, and hence their infinite subgroups cannot have property $T$ [NR97, NR98].

We apply Theorem 1.1 to obtain the following in Section 4 .

Theorem 1.2. Any countable group $Q$ embeds in Out $(N)$ for some group $N$ with property $T$.

In particular, there exists a group $N$ with property $T$ such that $\operatorname{Out}(N)$ is infinite.

The motivation is that, as proven by Paulin [Pau91, if $H$ is word-hyperbolic and $|\operatorname{Out}(H)|=\infty$, then $H$ splits over an infinite cyclic group, and hence $H$ cannot have property $T$. The question of whether every group with property $T$ has a finite outer automorphism group belongs to the list of open problems mentioned in de la Harpe and Valette's classical book on property $T$ (dlHV89], p. 134), was raised again by Alain Valette in his mathscinet review of [Pau91, and later appeared in a problem list from the 2002 meeting on property $T$ at Oberwolfach.

It may be useful to remind the reader of the definition of Kazhdan's property $T$ (see the excellent dlHV89], or [Val04] for a more recent review). This is a property of linear unitary representations of a locally compact group $G$ in the Hilbert space $\mathcal{H}$. Let $\rho$ be a linear unitary representation of $G$ in $\mathcal{H}$ : It has an invariant vector if there exists a unitary $v \in \mathcal{H}$ such that, for all $g \in G$, we have $\rho(g) v=v$. It has almost invariant vectors if for any compact $K \subset G$, for every $\varepsilon>0$, there exists a unitary vector $v \in \mathcal{H}$ such that, for all $g \in K,\|\rho(g) v-v\| \leqslant \varepsilon$ (so $K$ "almost fixes" $v$ ). The group $G$ has property $T$ (or is a Kazhdan group) if any unitary representation of $G$ having almost invariant vectors has an invariant vector.

Finally, we use the tools we developed to obtain the following two examples in Sections 6 and 5 .

Theorem 1.3. There exists a Kazhdan group $G$ that is not Hopfian.

Theorem 1.4. There exists a Kazhdan group $G$ that is not coHopfian.

Various other attempts to augment Rips's construction have focused on strengthening the properties of $G$ when $Q$ is f.p. (e.g.: $G$ is $\pi_{1}$ of a negatively curved complex Wis98; $G$ is a residually finite $C^{\prime}\left(\frac{1}{6}\right)$ group Wis03; $G$ is a subgroup of a rightangled Artin group, so $G \subset S L_{n}(\mathbb{Z})[\mathrm{HW04}]$.

1.2. Random groups. One key ingredient of our construction is the use of random methods to provide examples of groups with particular properties. Random groups were introduced by Gromov, originally in Gro87] and more and more extensively in Gro93 and Gro03. The original motivation was and still is the study of "typical" properties of groups. But random groups now have applications. We refer to Ghy03 and Oll05 for a general discussion of random groups.

We indeed use a result of Gro03] (see also [Sil03]) to provide a presentation of a group with property $T$ satisfying the graphical small cancellation property. In Section 7 we include a standalone proof of the results we need from Gro03. 


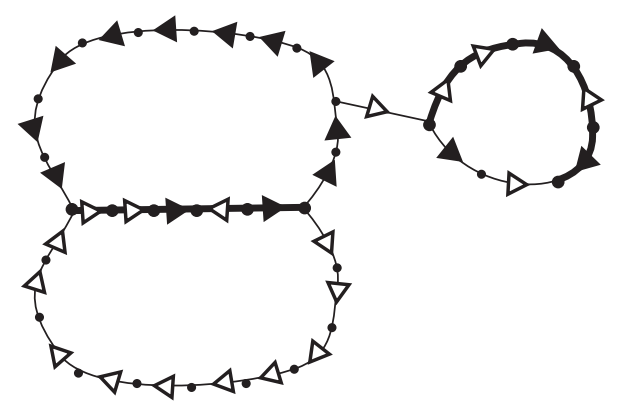

FiguRE 1.

Żuk showed that random groups with "enough" relators very probably have property $T\left[\dot{Z} u k 03\right.$. However, these groups never satisfy any kind of $C^{\prime}\left(\frac{1}{6}\right)$ small cancellation property, since the number of relators is too large (typically, having enough relators to get property $T$ creates pieces of relative length $>1 / 3$ between the relators). So we rely on the more elaborate construction of Gro03.

\section{2. $G r^{\prime}\left(\frac{1}{6}\right)$ GRAPHS}

2.1. Review of graphical $\alpha$-condition $G r^{\prime}(\alpha)$. Throughout this article, $B$ is a bouquet of $m \geqslant 2$ circles whose edges are directed and labelled, so that $m$ will be the number of generators of the group presentations we consider.

Let $\Gamma \rightarrow B$ be an immersed graph, and note that $\Gamma$ has an induced labelling. That $\Gamma$ immerses in $B$ simply denotes the fact that the words carried by paths immersed in $\Gamma$ are reduced.

By definition, the group $G$ presented by $\langle B \mid \Gamma\rangle$ has as generators the letters appearing on $B$, and relations consisting of all cycles appearing in $\Gamma$.

A piece $P$ in $\Gamma$ is an immersed path $P \rightarrow B$ which lifts to $\Gamma$ in more than one way. We refer the reader to Figure 1 for a graph with some pieces in it. The $a$ labels are indicated by a white triangle, and the $b$ labels are indicated by a black triangle. The bold path with label $a a b a^{-1} b$ is a piece since it appears twice in the graph as indicated by the bold paths. Other pieces include the paths $b^{10}$ and $a^{11}$.

Definition 2.1. We say $\Gamma \rightarrow B$ satisfies the graphical $\alpha$ condition $G r^{\prime}(\alpha)$ if for each piece $P$, and each cycle $C \rightarrow \Gamma$ such that $P \rightarrow \Gamma$ factors through $P \rightarrow C \uparrow \Gamma$, we have $|P|<\alpha|C|$.

The graphical $\alpha$ condition generalizes the usual $C^{\prime}(\alpha)$ : Let $\Gamma$ consist of the disjoint union of a set of cycles corresponding to the relators in a presentation. The graphical $\alpha$ condition is a case of a complicated but more general condition given by Gromov Gro03.

The condition $G r^{\prime}\left(\frac{1}{6}\right)$ implies that the group $G$ is torsion-free, word-hyperbolic whenever the graph is finite, and of dimension 2, just as the $C^{\prime}\left(\frac{1}{6}\right)$ condition Oll03a]. The group is non-elementary except in some explicit degenerate cases (a hyperbolic group is called elementary if it is finite or virtually $\mathbb{Z}$ ).

There is also a slightly stronger version of this condition, in which we demand that the size of the pieces be bounded not by $\alpha$ times the size of any cycle containing the piece, but by $\alpha$ times the girth of $\Gamma$ (recall the girth of a graph is the smallest 
length of a non-trivial closed path in it). We will sometimes directly prove this stronger version below, since it allows lighter notation.

A disc van Kampen diagram w.r.t. a graphical presentation is a van Kampen diagram, every 2-cell of which is labelled by a closed path immersed in $\Gamma$. It is reduced if, first, it is reduced in the ordinary sense and if moreover, for any two adjacent 2-cells, the boundary word of their union does not embed as a closed path in $\Gamma$ (otherwise, these two 2-cells can be replaced by a single one).

The following is easy to prove using the techniques in Oll03a.

Proposition 2.2. Let $\Gamma$ be a labelled graph satisfying $G r^{\prime}(\alpha)$ for $\alpha<\frac{1}{6}$. Let $D$ be a disc van Kampen diagram with respect to the presentation defined by $\Gamma$. Suppose that $D$ is reduced in the above sense. Then $D$ locally satisfies the ordinary $C^{\prime}(\alpha)$ small cancellation condition in the sense that two adjacent 2-cells of $D$ share a common path of length at most $\alpha$ times the infimum of their lengths.

Proof. Apply to $D$ the decomposition of Oll03a: Write $D$ as a union of maximal parts, the boundary word of which lifts to a cycle in $\Gamma$. Since $D$ is reduced, this decomposition is trivial, i.e. each single 2 -face of $D$ is such a maximal part (since otherwise we could replace all 2-cells in such a part by a single 2-cell with the same boundary word, which by definition lifts to a cycle in $\Gamma$ ). It is then proven in Oll03a that such maximal parts are in mutual $C^{\prime}(\alpha)$ small cancellation with the adjacent parts.

Of course it is not true that the set of all boundary words of all 2-cells of a reduced diagram $D$ satisfies the $C^{\prime}(\alpha)$ condition: Two non-adjacent 2-cells of $D$ may have boundary words which partially intersect once lifted to $\Gamma$. But what we have is exactly what is needed to entail all the usual consequences of small cancellation like isoperimetry and the Greendlinger lemma.

2.2. Producing more $G r^{\prime}\left(\frac{1}{6}\right)$ graphs. One useful feature of a presentation satisfying the ordinary $C^{\prime}\left(\frac{1}{6}\right)$ theory is that, provided that the relations are not "too dense" in a certain sense, more relations can be added to the presentation without violating the $C^{\prime}\left(\frac{1}{6}\right)$ condition.

In this subsection, we describe conditions on a $G r^{\prime}(\alpha)$ presentation such that additional relations can be added.

Proposition 2.3. Let $\Gamma \rightarrow B$ satisfy the $G r^{\prime}(\alpha)$ condition and suppose there is an immersed path $W \rightarrow B$ such that $1 \leqslant|W|<\frac{\alpha}{2} \operatorname{girth}(\Gamma)-1$, and $W$ does not lift to $\Gamma$.

Then there is a set of closed immersed paths $C_{i} \rightarrow B: i \in \mathbb{N}$ such that the disjoint union $\Gamma^{\prime}=\Gamma \sqcup_{i \in \mathbb{N}} C_{i} \rightarrow B$ satisfies the $G r^{\prime}(\alpha)$ condition.

Proof. We first form an immersed labelled graph $A \rightarrow B$ as follows: Let $D$ be the radius-2 ball at the basepoint of the universal cover $\tilde{B}$, and attach two copies $W_{x}$ and $W_{y}$ of the arc $W$ along four distinct leaves of $D$ as in Figure 2, (This can always be done avoiding the inverses of the initial and final letter of $W$, so that $D$ immerses in $B$.) Finally, we remove the finite trees that remain.

Observe that any path $P \leftrightarrow B$ that lifts to both $A$ and $\Gamma$ satisfies $|P|<$ $\alpha \operatorname{girth}(\Gamma)$. Indeed, if $P$ lifts to $\Gamma$, then $P$ cannot contain $W_{x}$ or $W_{y}$ as a subpath, and hence $P=U_{1} U_{2} U_{3}$, where $U_{1}$ and $U_{3}$ are proper initial or terminal subpaths of a $W$-arc, and $U_{2}$ is a path in $D$, so $|P| \leqslant\left|U_{1}\right|+\left|U_{2}\right|+\left|U_{3}\right| \leqslant(|W|-1)+4+(|W|-1)=$ $2|W|+2=2(|W|+1)<2 \frac{\alpha}{2} \operatorname{girth}(\Gamma)=\alpha \operatorname{girth}(\Gamma)$. 

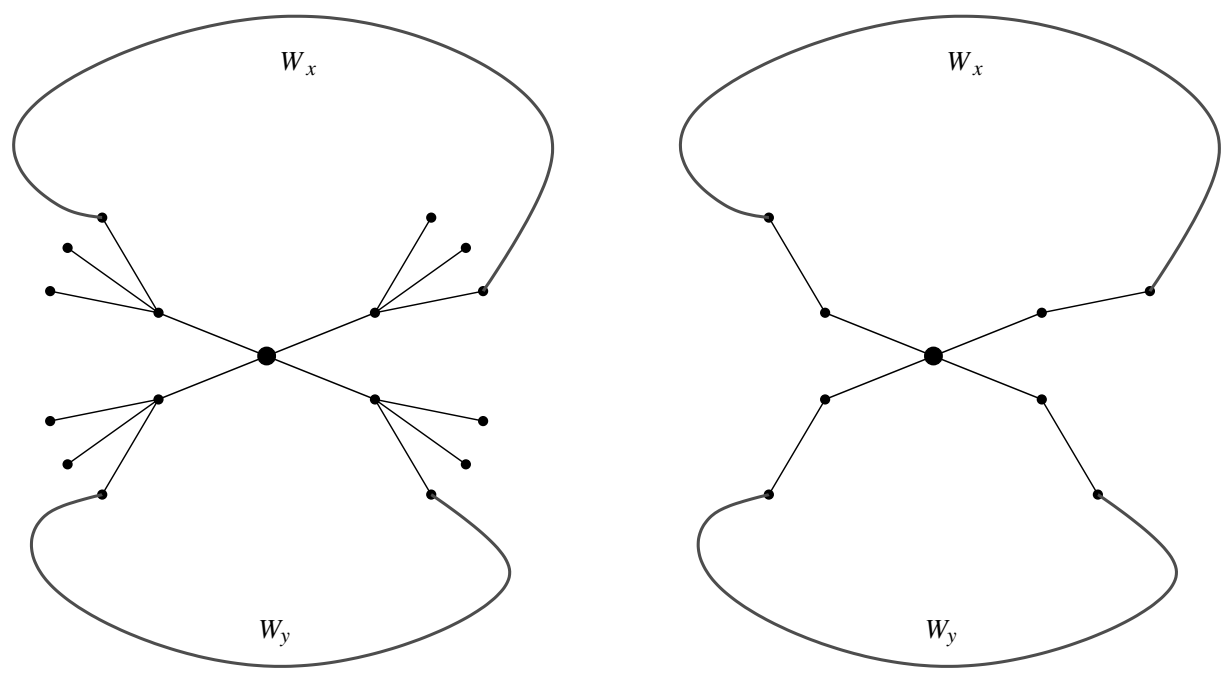

FIGURE 2 .

Now let $x$ and $y$ be arbitrary labels. To any reduced word $w$ in the letters $x^{ \pm 1}$ and $y^{ \pm 1}$ we can associate an immersed closed path $\varphi(w)$ in $A$ by sending $x$ to the based path in $A$ containing $W_{x}$, and similarly for $y$.

Now for each $i \in \mathbb{N}$, let $c_{i}$ denote the word $x y^{1000 i+1} x y^{1000 i+2} \cdots x y^{1000 i+999}$. It is easily verified that for large enough values of 1000 , the set of words $\left\langle x, y \mid c_{i}: i \in \mathbb{N}\right\rangle$ satisfies the $C^{\prime}\left(\frac{\alpha}{2}\right)$ condition.

Let $C_{i} \leftrightarrow \rightarrow B$ denote the corresponding closed immersed cycle $\varphi\left(c_{i}\right)$. Pieces in $\bigsqcup C_{i}$ are easily bounded in terms of pieces in $\left\langle x, y \mid c_{i}(i \in \mathbb{N})\right\rangle$, so that $\bigsqcup C_{i}$ satisfies the $G r^{\prime}(\alpha)$ (actually $\left.C^{\prime}(\alpha)\right)$ condition.

Finally $\Gamma^{\prime}=\Gamma \sqcup_{i \in \mathbb{N}} C_{i}$ satisfies the $G r^{\prime}(\alpha)$ condition since pieces that lift twice to $\Gamma$ are bounded by assumption, and we have just bounded pieces that lift to $\Gamma$ and to some $C_{i}$, and pieces that lift to some $C_{i}$ and some $C_{j}$.

Remark 2.4. The missing word condition in $\Gamma$ ensures that the group presented by $\Gamma$ is non-elementary. Indeed, the group presented by $\Gamma \sqcup_{i} C_{i}$ has infinite Euler characteristic (it is of dimension 2) and is thus non-elementary, so a fortiori the group presented by $\Gamma$ is non-elementary.

\section{The T Rips CONSTRUCtion}

Let us now turn to the proof of the main theorem of this article. We use an intermediate construction due to Gromov.

Proposition 3.1. There exists a finite graph $\Gamma$ that immerses in a bouquet $B$ of two circles such that:

(1) The group presented by $\langle B \mid \Gamma\rangle$ has property $T$.

(2) $\Gamma \leftrightarrow B$ satisfies the $G r^{\prime}\left(\frac{1}{12}\right)$ condition.

(3) There is a path $W \rightarrow B$ with $1 \leqslant|W|<\frac{1}{24} \operatorname{girth}(\Gamma)-1$, and $W$ does not lift to $\Gamma$.

(4) $\Gamma$ has arbitrarily large girth.

A proof of this is included in Section 7. 
Theorem 1.1, For each countable group $Q$, there is a short exact sequence $1 \rightarrow$ $N \rightarrow G \rightarrow Q \rightarrow 1$ such that

(1) $G$ is torsion-free,

(2) $G$ has a graphical $\frac{1}{6}$ presentation,

(3) $N$ has property $T$ and is non-trivial, and

(4) moreover, $G$ is finitely generated if $Q$ is, and finitely presented if $Q$ is.

Proof. Let $Q$ be given by the following presentation:

$$
\left\langle q_{i}: i \in I \mid R_{j}: j \in J\right\rangle .
$$

Let $\Gamma \leftrightarrow B$ be a graph provided by Proposition 3.1, where the edges of $B$ are labelled by $x$ and $y$. Let $\Gamma^{\prime}=\Gamma \sqcup_{n} C_{n}$ be as in Proposition 2.3 with $\alpha=1 / 12$.

The presentation for $G$ will be the following:

$$
\begin{aligned}
\left\langle x, y, q_{i}(i \in I)\right| \Gamma, & \\
x^{q_{i}}=X_{i+}, x^{q_{i}^{-1}}=X_{i-}, y^{q_{i}}=Y_{i+}, y^{q_{i}^{-1}}=Y_{i-} & (i \in I), \\
& \left.R_{j}=W_{j}(j \in J)\right\rangle,
\end{aligned}
$$

where superscripts denote conjugation, and where the $X_{i+}, X_{i-}, Y_{i+}, Y_{i-}$, and $W_{j}$ are equal to paths corresponding to distinct $C_{n}$ cycles of $\Gamma^{\prime},\left|W_{j}\right|>12\left|R_{j}\right|$ for each $j \in J$, and $\left|X_{i \pm}\right|>36,\left|Y_{i \pm}\right|>36$ for each $i \in I$.

The $\frac{1}{6}$ condition follows easily. Let us check, for example, that there is no $\frac{1}{6}$-piece between $\Gamma$ and the relation $x^{q_{i}}=X_{i+}$. Since the $q_{i}$ 's do not appear as labels on $\Gamma$, any such $\frac{1}{6}$-piece would be either $x$ or a subword of $X_{i+}$. The former is ruled out since $\operatorname{girth}(\Gamma)>6$. The latter would provide a piece between $\Gamma$ and $X_{i+}$ (which is one of the $C_{n}$ 's); such a piece is by assumption of length at most $\frac{1}{12}\left|X_{i+}\right|$, which in turn is less than $\frac{1}{6}\left|x^{q_{i}}=X_{i+}\right|$ as needed. The other cases are treated similarly.

Now $N$ is the subgroup of $G$ generated by $x$ and $y$. It is normal by construction of the presentation of $G$. Note that $N$ has property $T$ since it is a quotient of $\langle x, y \mid \Gamma\rangle$ which has property $T$ by choice of $\Gamma$.

Finally, $N$ is non-trivial: Indeed, we can pick some cycle $C_{n}$ which is a word in $x, y$ and which will be in small cancellation with the rest of the presentation. This provides a word in $x$ and $y$ which is not trivial in the group.

\section{KAZHDAN GROUPS WITH INFINITE OUTER AUTOMORPHISM GROUP}

Theorem 1.2. Any countable group $Q$ embeds in $\operatorname{Out}(N)$ for some group $N$ with property $T$.

In particular, there exists a group $N$ with property $T$ such that $\operatorname{Out}(N)$ is infinite.

Proof. For $1 \rightarrow N \rightarrow G \rightarrow Q \rightarrow 1$, the group $G$ acts by inner automorphisms on itself, so we have a homomorphism $G \rightarrow \operatorname{Aut}(N)$, and $N$ obviously maps to $\operatorname{Inn}(N)$ so there is an induced homomorphism $Q=G / N \rightarrow \operatorname{Out}(N)$. Elements in the kernel of $Q \rightarrow \operatorname{Out}(N)$ are represented by elements $g \in G$ such that $m^{g}=m^{n}$ for some $n \in N$ and all $m \in N$. Thus $g n^{-1}$ centralizes $N$.

First suppose that $Q$ is finitely presented, so that $G$ is as well.

In this case $N$ is a non-elementary subgroup of the torsion-free word-hyperbolic group $G$, and hence $N$ has a trivial centralizer. Indeed, $N$ must contain a rank 2 free subgroup $\left\langle n_{1}, n_{2}\right\rangle$ (see GdlH90, p. 157). If a non-trivial element $c$ centralizes $N$, then $\left\langle c, n_{1}\right\rangle$ and $\left\langle c, n_{2}\right\rangle$ are both abelian, and hence infinite cyclic since $G$ cannot 
contain a copy of $\mathbb{Z}^{2}$. Thus $n_{1}^{m_{1}}=c^{p_{1}}$ and $n_{2}^{m_{2}}=c^{p_{2}}$ for some $p_{i}, m_{i} \neq 0$. But then $n_{1}^{m_{1}}$ commutes with $n_{2}^{m_{2}}$, which is impossible.

Since the centralizer of $N$ is trivial, we have $g n^{-1}=1$, so $g \in N$, and hence $Q \rightarrow \operatorname{Out}(N)$ is injective.

The case when $Q$ is not finitely presented reduces back to the previous one: Indeed, suppose that some element $g$ of $G$ lies in the centralizer of $N$. This is equivalent to stating that $g$ commutes with $x$ and $y$. But $g$ can be written as a product of finitely many generators, and similarly the relations $[g, x]=1$ and $[g, y]=1$ are consequences of only finitely many relators, so that $g$ still lies in the centralizer of $N$ in a finite subpresentation of the presentation of $G$.

Remark 4.1. By adding some additional relations to $N$, the above argument was used in $[\mathrm{BW}$ to show that every countable group $Q$ appears as $\operatorname{Out}(N)$ for some f.g. $N$, and that every f.p. $Q$ appears as $\operatorname{Out}(N)$, where $N$ is f.g. and residually finite (but property $T$ did not appear there).

It appears likely that a more careful analysis along those lines would show that every countable group arises as $\operatorname{Out}(N)$ where $N$ has property $T$.

\section{A Kazhdan group that is not COHopfian}

Theorem 1.4. There exists a Kazhdan group that is not coHopfian.

Proof. Consider the group

$$
G=\left\langle a, b, t \mid \Gamma, a^{t}=\varphi(a), b^{t}=\varphi(b)\right\rangle,
$$

where $\varphi(a)$ and $\varphi(b)$ are chosen so that $\Gamma \sqcup \varphi(a) \sqcup \varphi(b)$ satisfies $G r^{\prime}\left(\frac{1}{6}\right)$ and $|\varphi(a)|>3$, $|\varphi(b)|>3$. (This is in fact a subpresentation of the presentation (1) used in the proof of Theorem 1.1.)

Clearly, the subgroup $K=\langle a, b\rangle$ is a Kazhdan group since it is a quotient of $\langle a, b \mid \Gamma\rangle$.

The map $K \rightarrow K$ induced by $\varphi$ is clearly well-defined and injective since it arises from conjugation in the larger group $G$.

We will now show that $\varphi$ is not surjective by verifying that $a \notin\langle\varphi(a), \varphi(b)\rangle$.

We argue by contradiction: Suppose that $a$ is equal in $G$ to a word $W(\varphi(a), \varphi(b))$ in $\varphi(a)$ and $\varphi(b)$; we can choose $W$ such that the disc diagram expressing this equality in the presentation for $G$ has minimal area among all such choices. Note that since $D$ is reduced and $G$ is $G r^{\prime}\left(\frac{1}{6}\right), D$ is a diagram satisfying the ordinary $C^{\prime}\left(\frac{1}{6}\right)$ condition in the sense of Proposition 2.2.

By Greendlinger's Lemma (after ignoring trees possibly attached to $\partial D$ ) either $D$ is a single 2-cell, or $D$ has at least two 2-cells whose outer paths are the majority of their boundaries.

The first possibility is excluded by consideration of the presentation for $G$. In the second case, one such 2-cell $R$ has outerpath $Q$ not containing the special $a$-edge in $\partial D$, as illustrated on the left in Figure 3 ,

The boundary word of the 2-cell $R$ cannot be a word immersing in $\Gamma$. Indeed, since it has more than half its length on the boundary of $D$ and this boundary bears a word in $\varphi(a)$ and $\varphi(b)$, this would contradict the small cancellation property of $\Gamma \sqcup \varphi(a) \sqcup \varphi(b)$. So $R$ is a 2-cell expressing the equality $a^{t}=\varphi(a)$ or $b^{t}=\varphi(b)$. Moreover, since $t$ does not appear on the boundary of $D$, the side of $R$ on the boundary is the $\varphi$-side. 

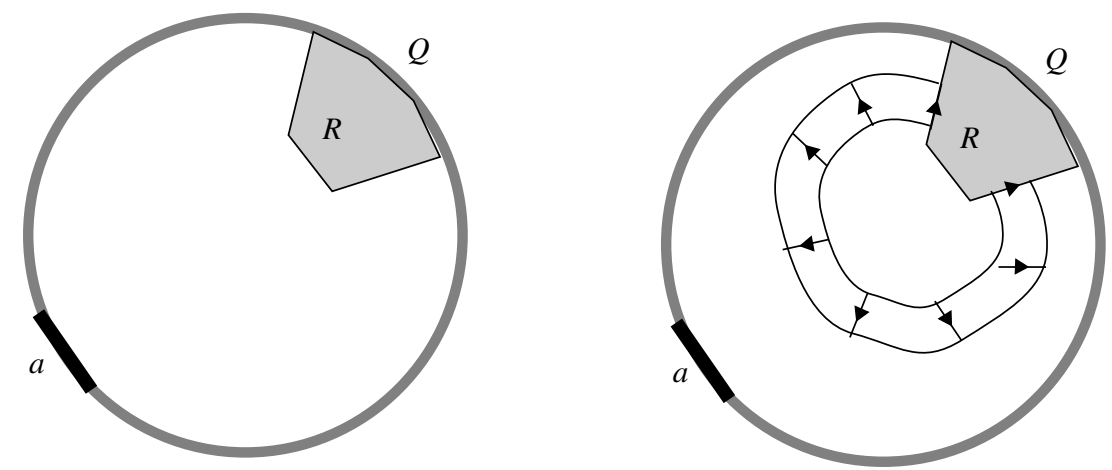

FiguRE 3.

Since $t \notin \partial D$, we can find a $t$-annulus containing $R$ as illustrated in the center of Figure 3, The $t$-edges in the figure are labelled by black triangles.

We now produce a new diagram $D^{\prime}$ with $\operatorname{Area}\left(D^{\prime}\right)<\operatorname{Area}(D)$. We do this by travelling around the $t$-annulus as on the right in Figure 3 ,

Observe that the small cancellation property implies that an edge in the $\varphi(a) \subset$ $\partial R$ or $\varphi(b) \subset \partial R$ lines up with an edge in some $\varphi(a)$ or $\varphi(b)$ in $\partial D$, and at exactly the same position. So if the $\varphi(a)$ or $\varphi(b)$ of $\partial R$ is not wholly contained in $\partial D$, after removing $R$ the words on the paths from $\partial D$ to the $t$-edges of $R$ will cancel with corresponding subwords of $\varphi(a)$ and $\varphi(b)$ lying in the remaining part of $\partial D$.

This implies that, after removing the annulus, the boundary of $D^{\prime}$ is labelled (maybe after folding) by a word of the form $a=W^{\prime}(\varphi(a), \varphi(b))$. But this is a contradiction since $D$ was assumed to be minimal.

(Note that $D^{\prime}$ might touch the special $a$-edge, and $D^{\prime}$ might have some extra singular edges.)

\section{A KaZHDAN GROUP THAT IS NOT HOPFian}

Definition 6.1. Let $B$ be a bouquet of circles, and let $\varphi: B \rightarrow B$. Let $A \rightarrow B$ be a map of graphs. Then we let $\varphi(A) \rightarrow B$ be the new map of graphs where $\varphi(A)$ is obtained from $A$ by substituting an $\operatorname{arc} \varphi(e)$ for each edge $e$ of $A$. That is, we replace the label on each edge of $A$ by its image under $\varphi$.

Lemma 6.2. Let $\Delta$ be a labelled graph satisfying $G r^{\prime}(\alpha)$ and $\alpha \operatorname{girth}(\Delta) \geqslant 1$. Suppose there is a path $P \rightarrow \Delta$ such that the edges in $P$ all bear the same label a and such that $P$ factors through a closed path $P \leftrightarrow C \rightarrow \Delta$. Then $|P|<2 \alpha|C|$.

Note that the assumption $\operatorname{girth}(\Delta) \geqslant 1 / \alpha$ is not very strong: If $\alpha \operatorname{girth}(\Delta) \leqslant 1$, then a single letter can constitute a piece, which can result in various oddities. This lemma is false for trivial reasons if we remove this girth assumption: When $\operatorname{girth}(\Delta)=1$ there are arbitrarily long homogeneous paths, though $G r^{\prime}(0)$ may be satisfied.

Proof. First, let us treat the trivial case when there is a length-1 loop bearing label $a$ : This implies $\operatorname{girth}(\Delta)=1$, so $\alpha=1$ and the equality to show is trivial. The case $|P|=1$ is trivial as well.

Second, suppose that there is no length-1 loop. Let $P$ be a path labelled by $a^{s}$ with $s \geqslant 2$. Then the two paths labelled by $a^{s-1}$ obtained by removing the first 
and last edge of $P$ respectively constitute a piece, and so we have $s-1<\alpha|C|$ so that $|P|=s<\alpha|C|+1 \leqslant \alpha(|C|+\operatorname{girth}(\Delta)) \leqslant 2 \alpha|C|$.

Lemma 6.3. Let $\Delta$ be a labelled graph satisfying $\operatorname{Gr}^{\prime}(\alpha)$ with $\alpha \operatorname{girth}(\Delta) \geqslant 1$, and let $\varphi: B \rightarrow B$ be induced by $a \mapsto a^{n}$ and $b \mapsto b^{n}$ for some $n \geqslant 1$. Then, for any $k \in \mathbb{N}, \varphi^{k}(\Delta)$ satisfies $\operatorname{Gr}^{\prime}(2 \alpha)$.

(Once more the girth assumption discards some degenerate cases when a single edge can make a piece.)

Proof. The reader should think of $\varphi^{k}(\Delta)$ as the $n^{k}$-subdivision of $\Delta$ where each $a$-edge is replaced by an arc of $n^{k} a$-edges and likewise for $b$-edges.

We begin by considering a homogeneous piece $P=a^{r}$ (or $P=b^{r}$, which is similar) occurring in some cycle $C \leftrightarrow \varphi^{k}(\Delta)$. Then $P$ is a subpath of a path $\varphi^{k}\left(P^{\prime}\right)$, where $P^{\prime}=a^{r^{\prime}}$ is a path in $\Delta$ and $P^{\prime}$ occurs in a cycle $C^{\prime}$ corresponding to $C$.

By the previous lemma, $\left|P^{\prime}\right|=r^{\prime}<2 \alpha\left|C^{\prime}\right|$ and so $|P|=r<2 \alpha n^{k}\left|C^{\prime}\right|=2 \alpha|C|$.

We now consider the general case where $P$ contains both $a$ and $b$ letters. We may assume that $P$ is a maximal piece, in which case $P=W\left(a^{n^{k}}, b^{n^{k}}\right)$, where $P^{\prime}=W(a, b)$ is itself a corresponding piece in $\Delta$. Everything scales by $n^{k}$, i.e. $|P|=n^{k}\left|P^{\prime}\right|<n^{k} \alpha\left|C^{\prime}\right|=\alpha|C|$.

Lemma 6.4. Let $\Delta$ satisfy $G r^{\prime}(\alpha)$ and suppose that $\operatorname{girth}(\Delta)>1 / \alpha$. Let $n$ satisfy $n>s$ where $s$ is the maximal length of a path $a^{s}$ or $b^{s}$ lifting to $\Delta$. Let $\varphi: B \rightarrow B$ be induced by $a \mapsto a^{n}$ and $b \mapsto b^{n}$. Then $\bigsqcup_{k \geqslant 0} \varphi^{k}(\Delta)$ satisfies $G^{\prime}(8 \alpha)$.

Note that $s=\infty$ implies either $\operatorname{girth}(\Delta)=1$ (which is excluded by assumption) or $\alpha=1$ (by removing the first and last letter of an arbitrarily long $a^{s}$-path) in which case the affirmation is void. So we can suppose $s<\infty$.

Proof. First, by the previous lemma, each $\varphi^{k}(\Delta)$ itself satisfies $G r^{\prime}(2 \alpha)$.

We now consider a piece $P$ between $\Delta$ and $\varphi^{k}(\Delta)$. Either $P \uparrow \varphi^{k}(\Delta)$ is contained in two subdivided edges of $\varphi^{k}(\Delta)$ so $|P|<2 n^{k}$, or $P$ contains an entire subdivided edge and hence an $a^{n^{k}}$ (or $b^{n^{k}}$ ) subpath.

In the latter case when $P$ contains an $a^{n^{k}}$ or $b^{n^{k}}$ subpath, since $P \hookrightarrow \Delta$ is a path in $\Delta$ then $n^{k}$ is at most the maximal length of an $a$-path or $b$-path in $\Delta$. But by hypothesis on $n$, this maximal length is bounded by $n$, and so $n^{k}<n$ which is impossible for $k \geqslant 1$.

In the former case, $P$ is the product of at most two homogeneous paths (i.e. $a$-paths or $b$-paths), one of which has length $\geqslant \frac{1}{2}|P|$. Thus by Lemma $6.2, \frac{1}{2}|P|<$ $2 \alpha|C|$ for any cycle $C$ in $\Delta$ containing $P$. So $|P|<4 \alpha|C|$ and $P$ cannot be a $4 \alpha-$ piece in $\Delta$. Besides, suppose that $P$ is included in a cycle $C$ immersed in $\varphi^{k}(\Delta)$. Since $|P|<2 n^{k}$ and $|C| \geqslant \operatorname{girth} \varphi^{k}(\Delta)=n^{k} \operatorname{girth}(\Delta) \geqslant n^{k} / \alpha$ by assumption, $P$ cannot consitute a $2 \alpha$-piece in $\varphi^{k}(\Delta)$ either. (Note that the constant 4 is this reasoning is optimal: Consider for $\Delta$ a circle of length 100 containing $a a b b$ at one place, and some garbage for the rest; take $\alpha=(1+\varepsilon) / 100$ so that the two $a$ 's do not form a piece. Then $\varphi(\Delta)$ contains some $a a b b$ as well, so that this word constitutes a $4 / 100$-piece in $\Delta \sqcup \varphi(\Delta)$.)

Finally, we consider pieces between $\varphi^{k}(\Delta)$ and $\varphi^{k^{\prime}}(\Delta)$ where we can suppose $k^{\prime}>k$. We have just proved that $\Delta \sqcup \varphi^{k^{\prime}-k}(\Delta)$ satisfies $G r^{\prime}(4 \alpha)$. We now apply Lemma 6.3 to see that $\varphi^{k}(\Delta) \sqcup \varphi^{k^{\prime}}(\Delta)=\varphi^{k}\left(\Delta \sqcup \varphi^{k^{\prime}-k}(\Delta)\right)$ satisfies $G r^{\prime}(8 \alpha)$. 
Remark 6.5. A generalization of Lemma 6.4 should hold with $\varphi(a)$ and $\varphi(b)$ appropriate small cancellation words instead of $a^{n}$ and $b^{n}$.

Theorem 1.3. There exists a Kazhdan group that is not Hopfian.

Proof. Let $G$ have the following presentation:

$$
\left\langle a, b \mid \varphi^{i}(\Gamma), \varphi^{i}\left(a \varphi\left(C_{1}\right)\right), \varphi^{i}\left(b \varphi\left(C_{2}\right)\right), \varphi^{i}\left(\varphi\left(C_{3}\right)\right)(i \geqslant 0)\right\rangle,
$$

where

(1) $\Gamma \sqcup C_{1} \sqcup C_{2} \sqcup C_{3}$ satisfies the $G r^{\prime}(\alpha)$ condition with $\alpha=1 / 2000\left(C_{1}, C_{2}\right.$ and $C_{3}$ arise from Proposition 2.3$)$;

(2) $\varphi$ is defined by $\varphi(a)=a^{n}$ and $\varphi(b)=b^{n}$, for some $n$ greater than the maximal length of an $a$-word or $b$-word in $\Gamma \sqcup C_{1} \sqcup C_{2} \sqcup C_{3}$;

(3) $\operatorname{girth}\left(\Gamma \sqcup C_{1} \sqcup C_{2} \sqcup C_{3}\right) \geqslant 2000$.

Let $\Delta_{0}=\bigsqcup_{k \geqslant 0} \varphi^{k}\left(\Gamma \sqcup C_{1} \sqcup C_{2} \sqcup C_{3}\right)$. By Lemma 6.4, this labelled graph satisfies $G r^{\prime}(8 \alpha)$. As a subgraph of $\Delta_{0}$, the graph $\Delta=\Gamma \sqcup \varphi\left(C_{1}\right) \sqcup \varphi\left(C_{2}\right) \sqcup C_{3}$ satisfies $G r^{\prime}(8 \alpha)$ as well.

We now prove that $\Delta^{\prime}=\Gamma \sqcup a \varphi\left(C_{1}\right) \sqcup b \varphi\left(C_{2}\right) \sqcup C_{3}$ is $G r^{\prime}(26 \alpha)$. Let $P$ be a piece involving the new $a$-edge or the new $b$-edge. Observe that $P=P_{1} a P_{2}$ (or $P_{1} b P_{2}$ ). Note that a new $b$ (or new $a$ ) may lie in at most one of $P_{1}$ or $P_{2}$. Thus $P$ is the concatenation of at most 3 pieces in $\Delta$ together with the new $a$ and possibly the new $b$. Consequently for any cycle $C$ containing $P$ we have $|P|<24 \alpha|C|+2 \leqslant 26 \alpha\left|C^{\prime}\right|$ where we have used the hypothesis that $\alpha$ girth $\geqslant 1$.

We now apply Lemma 6.4 to see that $\Omega=\bigsqcup_{k \geqslant 0} \varphi^{k}\left(\Delta^{\prime}\right)$ satisfies $G r^{\prime}(208 \alpha)$, and so does the presentation for $G$ which is a subset of $\Omega$.

Now $\varphi$ obviously sends relations to relations and thus induces a well-defined map in $G$. This map is surjective since $a \varphi\left(C_{1}\right)={ }_{G} 1$ and $b \varphi\left(C_{2}\right)={ }_{G} 1$.

Finally $\varphi$ is not injective since $\varphi\left(C_{3}\right)=_{G} 1$ but $C_{3} \neq_{G} 1$. Indeed, $C_{3}$ is in small cancellation relative to the relators of $G$ since both are included in $\Omega$.

\section{A $T G r^{\prime}\left(\frac{1}{6}\right)$ GRAPH WITH A MISSING WORD}

A main point in this paper is the following, introduced by Gromov in Gro03.

Proposition 7.1. For each $\alpha>0$ and $\alpha^{\prime}>0$ there exists a finite graph $\Gamma$ that immerses in a bouquet $B$ of two circles such that:

(1) The group presented by $\langle B \mid \Gamma\rangle$ has property $T$.

(2) $\Gamma \rightarrow B$ satisfies the $G r^{\prime}(\alpha)$ condition.

(3) There is a path $W \rightarrow B$ with $1 \leqslant|W| \leqslant \alpha^{\prime} \operatorname{girth}(\Gamma)$ and $W$ does not lift to $\Gamma$.

Moreover, the girth of $\Gamma$ can be taken arbitrarily large.

This trivially implies Proposition 3.1. It also results from Remark 2.4 that the obtained group is non-trivial.

The goal of the introduction of such graphs in Gro03 was to construct a group whose Cayley graph contains a family of expanders, in relation with the BaumConnes conjecture (see also Ghy03 and Oll03b). Therein, the construction is done starting not only with a free group but with an arbitrary hyperbolic group (compare Oll04), so that it can be iterated in order to embed a whole family of graphs. 
Here we use this construction for purposes closer to combinatorial group theory. We do not need the full strength of the iterated construction; this section is devoted to the proof of the statements we need.

We will use the following fact, the credit of which can be shared between Lubotzky, Margulis, Phillips, Sarnack, Selberg. We refer to Lub94] (Theorem 7.4.3 referring to Theorem 7.3.12) or to [DSV03].

Proposition 7.2. For lots of $v \in \mathbb{N}$, there is a family of graphs $\Gamma_{i}: i \in \mathbb{N}$ such that the following hold:

(1) Each $\Gamma_{i}$ is regular of valence $v$.

(2) $\inf _{i} \lambda_{1}\left(\Gamma_{i}\right)>0$ where $\lambda_{1}$ denotes the smallest non-zero eigenvalue of the discrete Laplacian $\Delta$.

(3) $\operatorname{girth}\left(\Gamma_{i}\right) \longrightarrow \infty$.

(4) $\exists C$ such that $\operatorname{Diameter}\left(\Gamma_{i}\right) \leqslant C$ girth $\left(\Gamma_{i}\right)$ for all $i$.

"Lots of $v$ " means e.g. that this works at least for $v=p+1$ with $p \geqslant 3$ prime ([Lub94], paragraph 1.2 refers to other constructions). This is irrelevant for our purpose.

We are going to use random labellings of subdivisions of the graphs $\Gamma_{i}$. Subdividing amounts to labelling each edge with a long word rather than just one letter, so that the small cancellation condition is more easily satisfied.

That the diameter of the graph is bounded by a constant times the girth reflects the fact that there are "not too many" relations added (compare the density model of random groups in Gro93 or Oll04): This amounts to taking an arbitrarily small density.

To prove Proposition 7.1 we need two more propositions.

Proposition 7.3. Given $v \in \mathbb{N}, \lambda_{0}>0$ and an integer $j \geqslant 1$ there exists an explicit $g_{0}$ such that if $\Gamma$ is a graph with $\operatorname{girth}(\Gamma) \geqslant g_{0}, \lambda_{1}(\Gamma) \geqslant \lambda_{0}$ and every vertex of $\Gamma$ has valency between 3 and $v$, then the random group defined through a random labelling of the $j$-subdivision $\Gamma^{j}$ of $\Gamma$ will have property $T$, with probability tending to 1 as the size of $\Gamma$ tends to infinity.

This is proven in Sil03] (Corollary 2.19, where $d$ is our $v, k$ is our number of generators $m$, and $|V|$ the size of the graph; in this reference, $\lambda(\Gamma)$ denotes the largest eigenvalue not equal to 1 of the averaging operator $1-\Delta$, so that the inequalities between this $\lambda$ and the first non-zero eigenvalue of $\Delta$ are reversed).

In the next proposition and for the rest of this section, $\Gamma^{j}$ denotes the $j$ subdivision of (the edges of) the graph $\Gamma$.

Proposition 7.4. For any $v \in \mathbb{N}$, any $\alpha>0$ and $\alpha^{\prime}>0$, for any $C \geqslant 1$, there exists an integer $j_{0}$ such that for any $j \geqslant j_{0}$, for any graph $\Gamma$ satisfying the conditions:

(1) Each vertex of $\Gamma$ is of valence at most $v$,

(2) The girth of $\Gamma$ is $g$,

(3) Diameter $(\Gamma) \leqslant C g$ for all $i$ (hence $\Gamma$ is finite and connected),

then the following properties hold with probability tending to 1 as $g \rightarrow \infty$ :

(1) The folded graph $\overline{\Gamma^{j}}$ obtained by a random labelling of $\Gamma^{j}$ satisfies the $G r^{\prime}(\alpha)$ condition.

(2) There is a reduced word of length between 1 and $\alpha^{\prime}$ girth $\overline{\Gamma^{j}}$ not appearing on any path in $\overline{\Gamma^{j}}$. 
This will be proven in the next sections (a sketch of proof can also be found in Gro03]).

Let us now just gather Propositions 7.2, 7.3 and 7.4

Proof of Proposition 7.1. Let $\alpha$ be the small cancellation constant to be achieved.

Apply Proposition 7.2 with some $v \in \mathbb{N}$ to get an infinite family of graphs $\Gamma_{i}$; let $\lambda_{0}$ be the lower bound on the spectral gap so obtained, and let $C$ be as in this proposition. Let us denote by $\Gamma_{i(g)}$ the first graph in this family having girth at least $g$.

For the chosen $\alpha>0$, let $j$ and $g$ be large enough for the conclusions of Proposition 7.4 to hold when applied to $\Gamma_{i(g)}$. Let $g$ be still large enough (depending on $j$ ) so that the conclusions of Proposition 7.3 applied to this $j$ hold. This provides a graph satisfying the three requirements of Proposition 7.1 .

7.1. Some simple properties of random words. Recall $m \geqslant 2$ is the number of generators we use. We denote by $\|w\|$ the norm in the free group of the word $w$, that is, the length of the associated reduced word.

Hereafter $\theta$ is the gross cogrowth of the free group (we refer to the paragraph "Growth, cogrowth, and gross cogrowth" in Oll04 for basic properties). Basically, $\theta$ is the infimum of the real numbers so that the number of words of length $\ell$ which freely reduce to the trivial word is at most $(2 m)^{\theta \ell}$ for all $\ell \in \mathbb{N}$. In particular, the probability that a random walk in the free group comes back at its origin at time $\ell$ is at most $(2 m)^{-(1-\theta) \ell}$. Explicitly we have $(2 m)^{\theta}=2 \sqrt{2 m-1}$ [Kes59].

We state here some elementary properties having to deal with the behavior of reducing a random word. The first one is pretty intuitive.

Lemma 7.5. Let $W_{\ell}$ be a random word of length $\ell$ and let $\bar{W}_{\ell}$ be the associated reduced word. Then the law of $\bar{W}_{\ell}$ knowing its length $\left|\bar{W}_{\ell}\right|=\left\|W_{\ell}\right\|$ is the uniform law on all reduced words of this length.

Proof of the lemma. The group of automorphisms of the $2 m$-regular tree preserving some basepoint acts transitively on the points at a given distance from the basepoint and preserves the law of the random walk starting at this basepoint.

The following is proven in Oll04, Proposition 17.

Lemma 7.6. Let $W_{\ell}$ be a random word of length $\ell$. Then, for any $0 \leqslant L \leqslant \ell$ we have

$$
\operatorname{Pr}\left(\left\|W_{\ell}\right\| \leqslant L\right) \leqslant(2 m)^{-\ell(1-\theta)+\theta L} .
$$

Note that the exponent vanishes for $L=\frac{1-\theta}{\theta} \ell<\ell$ ( since $\theta>1 / 2$ ). A slightly different, asymptotically stronger version of this lemma is the following.

Lemma 7.7. Let $W_{\ell}$ be a random word of length $\ell$. Then, for any $L$ we have

$$
\operatorname{Pr}\left(\left\|W_{\ell}\right\| \leqslant L\right) \leqslant \sqrt{\ell \frac{2 m}{2 m-1}}(2 m)^{-(1-\theta) \ell}(2 m-1)^{L / 2} .
$$


Proof. Let $B_{\ell}$ be the ball of radius $\ell$ centered at $e$ in the free group. Let $p_{x}^{\ell}$ be the probability that $W_{\ell}=x$. We have

$$
\begin{aligned}
\mathbb{E}(2 m-1)^{-\frac{1}{2}\left\|W_{\ell}\right\|} & =\sum_{x \in B_{\ell}} p_{x}^{\ell}(2 m-1)^{-\frac{1}{2}\|x\|} \\
& \leqslant \sqrt{\sum_{x \in B_{\ell}}\left(p_{x}^{\ell}\right)^{2}} \sqrt{\sum_{x \in B_{\ell}}(2 m-1)^{-\|x\|}}
\end{aligned}
$$

by the Cauchy-Schwarz inequality. But $\sum_{x \in B_{\ell}}\left(p_{x}^{\ell}\right)^{2}$ is exactly the probability of return to $e$ at time $2 \ell$ of the random walk (condition by where it is at time $\ell$ ) which is at most $(2 m)^{-2(1-\theta) \ell}$. Besides, there are $(2 m)(2 m-1)^{k-1}$ elements of norm $k$ in $B_{\ell}$, so that $\sum_{x \in B_{\ell}}(2 m-1)^{-\|x\|}=\sum_{0 \leqslant k \leqslant \ell}(2 m)(2 m-1)^{k-1}(2 m-1)^{-k}=\ell \frac{2 m}{2 m-1}$. So we get

$$
\mathbb{E}(2 m-1)^{-\frac{1}{2}\left\|W_{\ell}\right\|} \leqslant \sqrt{\ell \frac{2 m}{2 m-1}}(2 m)^{-(1-\theta) \ell} .
$$

Now we simply apply the Markov inequality

$$
\begin{aligned}
\operatorname{Pr}\left(\left\|W_{\ell}\right\| \leqslant L\right) & =\operatorname{Pr}\left((2 m-1)^{-\frac{1}{2}\left\|W_{\ell}\right\|} \geqslant(2 m-1)^{-\frac{1}{2} L}\right) \\
& \leqslant(2 m-1)^{\frac{1}{2} L} \mathbb{E}(2 m-1)^{-\frac{1}{2}\left\|W_{\ell}\right\|}
\end{aligned}
$$

to get the conclusion.

7.2. Folding the labelled graph. Labelling a graph by plain random words does generally not result in a reduced labelling. Nevertheless, we can always fold the resulting labelled graph. Here we show that in the circumstances needed for our applications, this folding is a quasi-isometry. This will allow a transfer of the $G r^{\prime}$ small cancellation condition from the unfolded to the folded graph.

Proposition 7.8. For any $\beta>0$, for any $v \in \mathbb{N}$, and for any $C \geqslant 1$, there exists an integer $j_{0}$ such that for any $j \geqslant j_{0}$, for any graph $\Gamma$ satisfying the conditions:

(1) Vertices of $\Gamma$ are of valency at most $v$,

(2) Diameter $(\Gamma) \leqslant C g$ for all $i$, where $g$ is the girth of $\Gamma$,

then the folding map $\Gamma^{j} \rightarrow \overline{\Gamma^{j}}$ from a random labelling $\Gamma^{j} \rightarrow B$ to the associated reduced labelling $\overline{\Gamma^{j}} \rightarrow B$ is a $\left(\frac{\theta}{1-\theta}, \beta j g, g j\right)$ local quasi-isometry, with probability tending to 1 as $g \rightarrow \infty$.

We use the notation from GdlH90] for local quasi-isometries: An $(a, b, c)$ local quasi-isometry is a map $f$ such that whenever $d(x, y) \leqslant c$ we have $\frac{1}{a} d(x, y)-b \leqslant$ $d(f(x), f(y)) \leqslant a d(x, y)+b$. Here folding obviously decreases distances so that only the left inequality has to be checked.

Remark 7.9. Below we will make repeated use of the following: The number of paths of length $\ell$ in $\Gamma^{j}$ is at most $j^{2} v^{C g+\ell / j}$. Indeed, the number of points in $\Gamma$ is at most $v^{C g}$, and once a point is chosen the number of paths of length $k$ originating at it is at most $v^{k}$. Now specifying a path in the subdivision $\Gamma^{j}$ amounts to specifying a path in $\Gamma$ and giving two integers between 1 and $j$ to specify the exact endpoints.

Proof. Unwinding the definition of local quasi-isometries, we have to prove that any immersed path of length $\beta g j+\ell \leqslant g j$ in $\Gamma^{j}$ is mapped onto a path of length at least $\frac{1-\theta}{\theta} \ell$ in $\overline{\Gamma^{j}}$. 
By Remark 7.9, there are at most $j^{2} v^{C g+g}$ paths of length $g j$ in the subdivision $\Gamma^{j}$ of $\Gamma$. Fix such a path, of length say $\beta g j+\ell$.

Since the length of the immersed path is at most $g j=\operatorname{girth}\left(\Gamma^{j}\right)$, the path does not travel twice along the same edge. Consequently, the labels appearing on this path are all chosen independently. Then by Lemma 7.6, the probability that its length after folding is less than $\frac{1-\theta}{\theta} \ell$ is less than

$$
(2 m)^{-(1-\theta)(\ell+\beta g j)+\theta \frac{1-\theta}{\theta} \ell}=(2 m)^{-(1-\theta) \beta g j}
$$

for this particular path. Since the number of choices for the path is at most $j^{2} v^{C g+g}$, if $j$ is large enough depending on $C, \beta$ and $\theta$, namely if $v^{C+1}(2 m)^{-(1-\theta) \beta j}<1$, then the probability that there exists a path violating our local quasi-isometry property will tend to 0 as $g \rightarrow \infty$.

Corollary 7.10. In the same circumstances, the girth of $\overline{\Gamma^{j}}$ is at least $\frac{1-\theta}{\theta}-\beta$ times that of $\Gamma^{j}$.

Proof. Take a simple closed path $p$ in $\overline{\Gamma^{j}}$. It is the image of a non-null-homotopic closed path $q$ in $\Gamma^{j}$, whose length is by definition at least $g j=\operatorname{girth} \Gamma^{j}$. Let $q^{\prime}$ be the initial subpath of $q$ of length $g j$. We can apply the local quasi-isometry statement to $q^{\prime}$, showing that its image $p^{\prime}$ has length at least $\frac{1-\theta}{\theta} g j-\beta g j$, which is thus a lower bound on the length of $p$.

7.3. Pieces in the unfolded and folded graphs. Here we show that under the circumstances above, the probability to get a long piece in the folded graph is very small.

Suppose again that we are given a graph $\Gamma$ of degree at most $v$, of girth $g$ and of diameter at most $C g$. Consider its $j$-subdivision $\Gamma^{j}$ endowed with a random labelling and let $\overline{\Gamma^{j}}$ be the associated folded labelled graph.

Let $p, p^{\prime}$ be two immersed paths in $\overline{\Gamma^{j}}$. Let $q, q^{\prime}$ be some preimages in $\Gamma^{i}$ of $p, p^{\prime}$. If $p$ and $p^{\prime}$ are labelled by the same word, then $q$ and $q^{\prime}$ will be labelled by some freely equal words, so that pieces come from pieces.

Note that in a graph labelled by non-reduced words, there are some "trivial pieces", e.g. if some $a a^{-1}$ appears next to a word $w$, then $\left(w, a a^{-1} w\right)$ will be a piece. Such pieces disappear after folding the labelled graph; this is why we discard them in the following.

Proposition 7.11. Let $q, q^{\prime}$ be two immersed paths in a graph $\Delta$ of girth $g$. Suppose that $q$ and $q^{\prime}$ have length $\ell$ and $\ell^{\prime}$ respectively, with $\ell$ and $\ell^{\prime}$ at most $g / 2$. Endow $\Delta$ with a random labelling. Suppose that after folding the graph, the paths $q$ and $q^{\prime}$ are mapped to distinct paths. Then the probability that $q$ and $q^{\prime}$ are labelled by two freely equal words is at most

$$
C_{\ell, \ell^{\prime}}(2 m)^{-(1-\theta)\left(\ell+\ell^{\prime}\right)}
$$

where $C_{\ell, \ell^{\prime}}$ is a term growing subexponentially in $\ell+\ell^{\prime}$.

Proof. Let $w$ and $w^{\prime}$ be the words labelling $q$ and $q^{\prime}$, respectively.

First, assume that the images of $q$ and $q^{\prime}$ in $\Delta$ are disjoint. Then the letters making up $w$ and $w^{\prime}$ are chosen independently, and thus the word $w w^{\prime-1}$ is a plain random word. Thus is this case the proposition is just a rewriting of the definition of $\theta$. 


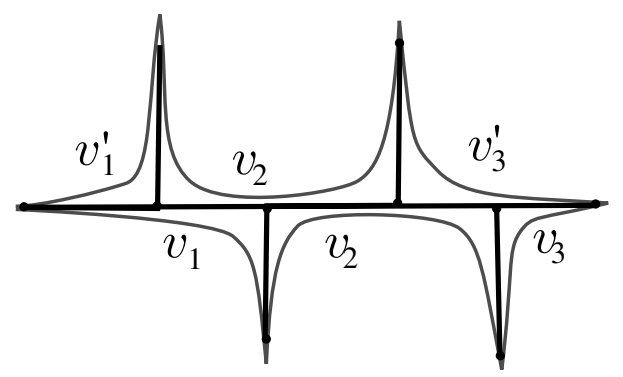

Figure 4.

Second, suppose that the paths do intersect in $\Delta$ : This results in lack of independence in the choice of the letters making up $w$ and $w^{\prime}$ (the same problem is treated in a slightly different setting in Oll04, section "Elimination of doublets"), which needs to be treated carefully. Since the length of these words is less than half the girth, the intersection in $\Delta$ is connected, and we can write $w=u_{1} u_{2} u_{3}$, $w^{\prime}=u_{1}^{\prime} u_{2} u_{3}^{\prime}$ where the $u_{i}$ 's are independently chosen random words (depending on relative orientation of $w$ and $w^{\prime}, u_{2}^{-1}$ rather than $u_{2}$ may appear in $\left.w^{\prime}\right)$. We can suppose that $u_{1}^{\prime} u_{1}^{-1}$ is not freely trivial: Otherwise the two paths start at the same point after folding, and so if $w=w^{\prime}$ we also have $u_{3}^{\prime} u_{3}^{-1}=e$ so that they also end at the same point after folding, but this is discarded by assumption. Likewise $u_{3}^{\prime} u_{3}^{-1}$ is not freely trivial.

Let $v_{1}, v_{2}, v_{3}, v_{1}^{\prime}, v_{3}^{\prime}$ be the reduced words freely equal to $u_{1}, u_{2}, \ldots$, respectively.

Lemma 7.5 tells us that the words $v_{1}, v_{2}, \ldots$ are random reduced words. Now let us draw a picture expressing the equality $v_{1} v_{2} v_{3}=v_{1}^{\prime} v_{2} v_{3}^{\prime}$ (Figure 4 ).

Note that the two copies of $v_{2}$ have to be shifted relatively to each other, otherwise this means that $u_{1}^{\prime} u_{1}^{-1}$ and $u_{3}^{\prime} u_{3}^{-1}$ are freely trivial.

Let $k$ be the length shared between the two copies of $v_{2}$. Now let us evaluate the probability of this situation knowing all the lengths of the words $v_{1}, v_{2}, \ldots$. Conditionally to their lengths, these words are uniformly chosen random reduced words by Lemma 7.5

We begin with the two copies of $v_{2}$ : Though they are not chosen independently, since we know that they are shifted, adding letter after letter we see that the probability that they can glue along a subpath of length $k$ is a most $1 /(2 m-1)^{k}$. Once $v_{2}$ is given, the words $v_{1}, v_{3}, v_{1}^{\prime}, v_{3}^{\prime}$ are all chosen independent of each other. The probability that they glue according to the picture is $1 /(2 m-1)^{L-k}$, where $L$ is the total length of the picture. So the overall probability of such a gluing is $1 /(2 m-1)^{L}$.

We obviously have $\ell+\ell^{\prime}=\left|v_{1}\right|+2\left|v_{2}\right|+\left|v_{3}\right|+\left|v_{1}^{\prime}\right|+\left|v_{3}^{\prime}\right|=2 L$. Now by Lemma 7.7 applied to all these words separately, the probability of achieving this value of $\left|v_{1}\right|+2\left|v_{2}\right|+\left|v_{3}\right|+\left|v_{1}^{\prime}\right|+\left|v_{3}^{\prime}\right|$ is less than

$$
\begin{aligned}
C_{\ell, \ell^{\prime}} & (2 m)^{-(1-\theta)\left(\left|u_{1}\right|+2\left|u_{2}\right|+\left|u_{3}\right|+\left|u_{1}^{\prime}\right|+\left|u_{3}^{\prime}\right|\right)}(2 m-1)^{\frac{1}{2} 2 L} \\
& =C_{\ell, \ell^{\prime}}(2 m)^{-(1-\theta)\left(\ell+\ell^{\prime}\right)}(2 m-1)^{L},
\end{aligned}
$$

where $C_{\ell, \ell^{\prime}}$ is a term growing subexponentially in $\ell+\ell^{\prime}$. 
So the overall probability of such a situation, taking into account the possibilities for $L$ between 0 and $\ell+\ell^{\prime}$, is at most

$$
\sum_{0 \leqslant L \leqslant \ell+\ell^{\prime}}(2 m-1)^{-L} C_{\ell+\ell^{\prime}}(2 m)^{-(1-\theta)\left(\ell+\ell^{\prime}\right)}(2 m-1)^{L}
$$

since we just proved above that $(2 m-1)^{-L}$ is an upper bound for the probability of the situation knowing $L$. But this is equal to $C_{\ell+\ell^{\prime}}^{\prime}(2 m)^{-(1-\theta)\left(\ell+\ell^{\prime}\right)}$, where $C_{\ell+\ell^{\prime}}^{\prime}$ is another term growing subexponentially in $\ell+\ell^{\prime}$.

We are now ready to prove Proposition 7.4 stating that the $G r^{\prime}(\alpha)$ condition is satisfied with overwhelming probability. In order to avoid heavy notation, we will directly prove the stronger variant of the $G r^{\prime}$ condition involving the girth instead of the length of cycles containing the pieces (see Section 2).

Proof of Proposition 7.4, small cancellation part. Since ruling out small pieces rules out larger pieces as well, it is enough to work for small $\alpha$.

Let $\bar{g}$ be the girth of $\overline{\Gamma^{j}}$. By Corollary 7.10 , we can assume that $\bar{g} \geqslant\left(\frac{1-\theta}{\theta}-\beta\right) g j$ with overwhelming probability, for arbitrarily small $\beta$.

Let $p, p^{\prime}$ be two distinct immersed paths in $\overline{\Gamma^{j}}$ forming a $\alpha$-piece; both $p$ and $p^{\prime}$ are of length $\alpha \bar{g}$. Let $q$ and $q^{\prime}$ be some immersed paths in $\Gamma^{j}$ mapping to $p$ and $p^{\prime}$.

Suppose that the length of $q$ (or $q^{\prime}$ ) is greater than $g j / 2$. By applying the local quasi-isometry property to an initial subpath of $q$ of length $g j / 2$ we get that the length of $p$ would be at least $\frac{1-\theta}{\theta} g j / 2-\beta g j$. But the length of $p$ is exactly $\alpha \bar{g} \leqslant \alpha g j$, so that if $\alpha$ and $\beta$ are taken small enough (depending on $\theta$ ) we get a contradiction. Hence, the length of $q$ is at most $g j / 2$, so that we are in a position to apply Proposition 7.11 .

The length of $q$ and $q^{\prime}$ is at least that of $p$ and $p^{\prime}$, namely $\alpha \bar{g}$, and since $\bar{g} \geqslant$ $\left(\frac{1-\theta}{\theta}-\beta\right) g j, q$ and $q^{\prime}$ form an $\alpha\left(\frac{1-\theta}{\theta}-\beta\right)$-piece in $\Gamma^{j}$. Now Proposition 7.11 states that for fixed $q$ and $q^{\prime}$ in $\Gamma^{j}$, the probability of this is at most

$$
C_{g j}(2 m)^{-(1-\theta) 2 g j \alpha\left(\frac{1-\theta}{\theta}-\beta\right),}
$$

where $C_{g j}$ is a subexponential term in $|q|+\left|q^{\prime}\right| \leqslant g j$.

By Remark 7.9, the number of choices for $q$ and $q^{\prime}$ is at most $j^{4} v^{(2 C+1) g}$. So the probability that one of these choices gives rise to a piece is at most

$$
j^{4} v^{(2 C+1) g} C_{g j}(2 m)^{-(1-\theta) 2 g j \alpha\left(\frac{1-\theta}{\theta}-\beta\right)} .
$$

Now, if $\beta$ is taken small enough (depending only on $\theta$ ) and if $j$ is taken large enough (depending on $\alpha, \theta$ and $C$ but not on $g$ ), namely if

$$
v^{2 C+1}(2 m)^{-(1-\theta) 2 j \alpha\left(\frac{1-\theta}{\theta}-\beta\right)}<1,
$$

then this tends to 0 when $g$ tends to infinity.

Proof of Proposition 7.4, missing word part. We now prove that for any $\alpha^{\prime}>0$, in the same circumstances, there exists a reduced word of length $\alpha^{\prime} \operatorname{girth}\left(\overline{\Gamma^{j}}\right)$ not appearing on any path in $\overline{\Gamma^{j}}$.

Let $p$ be a simple path of length $\alpha^{\prime} \bar{g}$ in $\overline{\Gamma^{j}}$. It is the image of some path $q$ in $\Gamma^{j}$ of length at least $\alpha^{\prime} \bar{g} \geqslant \alpha^{\prime}\left(\frac{1-\theta}{\theta}-\beta\right) g j$. But by Remark 7.9, the number of such paths in $\Gamma^{j}$ is at most $j^{2} v^{C g+\alpha^{\prime}\left(\frac{1-\theta}{\theta}-\beta\right) g}$, whereas the total number of reduced words of 
this length is at least $(2 m-1)^{\alpha^{\prime}\left(\frac{1-\theta}{\theta}-\beta\right) g j}$. So if $j$ is taken large enough (depending on $\alpha^{\prime}$ and $\theta$ but not on $g$ ), that is, if

$$
v^{C+\alpha^{\prime}\left(\frac{1-\theta}{\theta}-\beta\right)}<(2 m-1)^{\alpha^{\prime}\left(\frac{1-\theta}{\theta}-\beta\right) j},
$$

then the possible reduced words outnumber the paths in $\overline{\Gamma^{j}}$ when $g \rightarrow \infty$, so that there has to be a missing word.

\section{Problems}

Does there exist a finitely presented group $N$ with property $T$ such that $\operatorname{Out}(N)$ is infinite?

Let $Q$ be a f.p. group with property $T$. Does there exist word-hyperbolic $G$ with property $T$ and f.g. normal subgroup $N$ such that $Q=G / N$ ?

Do there exist f.p. Kazhdan groups which are not Hopfian or coHopfian?

Remark 8.1. Yves de Cornulier informed us after learning of our work that he can construct explicit f.p. Kazhdan groups with infinite order outer automorphisms Cor. His examples use lattices in Lie groups.

\section{ACKNOWLEDGEMENTS}

We would like to thank Yves de Cornulier and Frédéric Paulin for helpful comments on the text.

\section{REFERENCES}

[BW] Inna Bumagin and Daniel T. Wise, Every group is an outer automorphism group of a finitely generated group, J. Pure Appl. Algebra 200 (2005), no. 1-2, 137-147. MR 2142354 (2005m:20085)

[Cor] Yves de Cornulier, Finitely presentable, non-Hopfian groups with Kazhdan's property (T) and infinite outer automorphism group, To appear in Proc. Amer. Math. Soc.

[DSV03] Giuliana Davidoff, Peter Sarnak, and Alain Valette, Elementary number theory, group theory, and Ramanujan graphs, London Mathematical Society Student Texts, vol. 55, Cambridge University Press, Cambridge, 2003. MR1989434 (2004f:11001)

[dlHV89] Pierre de la Harpe and Alain Valette, La propriété (T) de Kazhdan pour les groupes localement compacts, Astérisque, no. 175, Soc. Math. Fr., 1989, with an appendix by M. Burger. MR1023471 (90m:22001)

[GdlH90] É. Ghys and P. de la Harpe (eds.), Sur les groupes hyperboliques d'après Mikhael Gromov, Progress in Mathematics, vol. 83, Birkhäuser Boston, Inc., Boston, MA, 1990, papers from the Swiss Seminar on Hyperbolic Groups held in Bern, 1988. MR1086648 (92f:53050)

[Ghy03] Étienne Ghys, Groupes aléatoires (d'après Misha Gromov,...), Astérisque (2004), no. 294, 173-204, Séminaire Bourbaki, Vol. 2003/2004, Exp. $916 . \quad$ MR2111644 (2005j:20049)

[Gro87] M. Gromov, Hyperbolic groups, Essays in group theory, Math. Sci. Res. Inst. Publ., vol. 8, Springer, New York, 1987, pp. 75-263. MR0919829 (89e:20070)

[Gro93] Asymptotic invariants of infinite groups, Geometric group theory, Vol. 2 (Sussex, 1991), Cambridge Univ. Press, Cambridge, 1993, pp. 1-295. MR1253544 (95m:20041)

[Gro03] , Random walk in random groups, Geom. Funct. Anal. 13 (2003), no. 1, 73-146. MR $1978492(2004 \mathrm{j}: 20088 \mathrm{a})$

[HW04] Frédéric Haglund and Daniel T. Wise, Special cube complexes, preprint, 2004.

[Kes59] Harry Kesten, Symmetric random walks on groups, Trans. Amer. Math. Soc. 92 (1959), 336-354. MR0109367 (22:253) 
[Lub94] Alexander Lubotzky, Discrete groups, expanding graphs and invariant measures, Progress in Mathematics, vol. 125, Birkhäuser Verlag, Basel, 1994, with an appendix by Jonathan D. Rogawski. MR1308046 (96g:22018)

[LS77] Roger C. Lyndon and Paul E. Schupp, Combinatorial group theory, Springer-Verlag, Berlin, 1977, Ergebnisse der Mathematik und ihrer Grenzgebiete, Band 89. MR0577064 $(58: 28182)$

[NR97] Graham Niblo and Lawrence Reeves, Groups acting on CAT(0) cube complexes, Geom. Topol. 1 (1997), approx. 7 pp. (electronic). MR.1432323 (98d:57005)

[NR98] Graham A. Niblo and Martin A. Roller, Groups acting on cubes and Kazhdan's property (T), Proc. Amer. Math. Soc. 126 (1998), no. 3, 693-699. MR.1459140 (98k:20058)

[Oll03a] Y. Ollivier, On a small cancellation theorem of Gromov, to appear in Bull. Belgian Math. Soc.

[Oll03b] — Cayley graphs containing expanders, after Gromov, expository manuscript, 2003.

[Oll04] Sharp phase transition theorems for hyperbolicity of random groups, GAFA, Geom. Funct. Anal. 14 (2004), no. 3, 595-679. MR2100673 (2005m:20101)

[Oll05] , A January 2005 invitation to random groups, Ensaios Matemáticos 10, Sociedade Brasileira de Matemática, Rio de Janeiro, 2005. MR2205306

[Pau91] Frédéric Paulin, Outer automorphisms of hyperbolic groups and small actions on $\mathbf{R}$ trees, Arboreal group theory (Berkeley, CA, 1988), Math. Sci. Res. Inst. Publ., vol. 19, Springer, New York, 1991, pp. 331-343. MR1105339 (92g:57003)

[Rip82] E. Rips, Subgroups of small cancellation groups, Bull. London Math. Soc. 14 (1982), no. 1, 45-47. MR0642423 (83c:20049)

[Sil03] L. Silberman, Addendum to "Random walk in random groups" by M. Gromov, GAFA, Geom. Funct. Anal. 13 (2003), no. 1, 147-177. MR1978493 (2004j:20088b)

[Val04] Alain Valette, Nouvelles approches de la propriété (T) de Kazhdan, Astérisque (2004), no. 294, 97-124, Séminaire Bourbaki, Vol. 2003/2004, Exp. 913. MR2111641 (2005j:22003)

[Wis98] Daniel T. Wise, Incoherent negatively curved groups, Proc. Amer. Math. Soc. 126 (1998), no. 4, 957-964. MR:1423338 (98f:20016)

[Wis03] _ A residually finite version of Rips's construction, Bull. London Math. Soc. 35 (2003), no. 1, 23-29. MR.1934427 (2003g:20047)

[Wis04] Daniel T. Wise, Cubulating small cancellation groups, GAFA, Geom. Funct. Anal. 14 (2004), no. 1, 150-214. MR2053602 (2005c:20069)

[Żuk03] A. Żuk, Property (T) and Kazhdan constants for discrete groups, Geom. Funct. Anal. 13 (2003), no. 3, 643-670. MR1995802 (2004m:20079)

CNRS, UMPA, École nORMALE SUPÉRIEURE DE Lyon, 46, ALlÉE D'ItALIE, 69364 Lyon CEDEX 7, FRANCE

E-mail address: yann.ollivier@umpa.ens-lyon.fr

Department of Mathematics, McGill University, Montréal, Québec, Canada H3A $2 \mathrm{~K} 6$

E-mail address: wise@math.mcgill.ca 